\title{
Automatic Web Spreadsheet Data Extraction
}

\author{
Zhe Chen \\ University of Michigan \\ Ann Arbor, MI 48109-2121 \\ chenzhe@umich.edu
}

\author{
Michael Cafarella \\ University of Michigan \\ Ann Arbor, Ml 48109-2121 \\ michjc@umich.edu
}

\begin{abstract}
Spreadsheets contain a huge amount of high-value data but do not observe a standard data model and thus are difficult to integrate. A large number of data integration tools exist, but they generally can only work on relational data. Existing systems for extracting relational data from spreadsheets are too labor intensive to support ad-hoc integration tasks, in which the correct extraction target is only learned during the course of user interaction.

This paper introduces a system that automatically extracts relational data from spreadsheets, thereby enabling relational spreadsheet integration. The resulting integrated relational data can be queried directly or can be translated into RDF triples. When compared to standard techniques for spreadsheet data extraction on a set of 100 random Web spreadsheets, the system reduces the amount of human labor by $72 \%$ to $92 \%$. In addition to the system design, we present the results of a general survey of more than 400,000 spreadsheets we downloaded from the Web, giving a novel view of how users organize their data in spreadsheets.
\end{abstract}

\section{INTRODUCTION}

Spreadsheets have become a critical data management tool $[3,20]$. They allow non-experts to perform tasks we traditionally associate with relational systems: selection, projection, sorting, etc. Spreadsheets make up some of the most expensive data available to us, because they have been constructed by hand by well-paid professionals. They are a standard tool for many researchers, scientists, and policymakers and have found especially wide adoption in financial and clinical research settings [15]. In short, spreadsheets make up an important dataset but live outside mainstream data management practices.

Spreadsheets often contain data that are roughly relational, but the schema is often designed for human consumption and entirely implicit. As a result, spreadsheets cannot benefit from society's huge investment in data management tools that work on relational databases. In par-

Permission to make digital or hard copies of all or part of this work for personal or classroom use is granted without fee provided that copies are not made or distributed for profit or commercial advantage and that copies bear this notice and the full citation on the first page. To copy otherwise, to republish, to post on servers or to redistribute to lists, requires prior specific permission and/or a fee.

SSW'13, August 30, 2013, Riva del Garda, Italy.

Copyright 2013 ACM 978-1-4503-2483-0/30/08 ...\$15.00. ticular, spreadsheets lack data integration operations. For example, it is easy to imagine an analyst who wants to combine a spreadsheet about company sales with a governmentproduced spreadsheet about economic performance to predict future sales. But in practice, the analyst would likely have to write custom code to integrate the two spreadsheets. If we could automate this burdensome integration procedure, users could make vastly more effective use of the spreadsheet data on the Web, intranets, and elsewhere. Given the high value of data stored in spreadsheets, such a system would likely have a large impact on the lives of analysisminded Web users.

Extracting relational data from spreadsheets would enable traditional data integration methods to unlock the latent value in spreadsheet data. Recent studies $[1,2,8,11]$ attempted to transform spreadsheet data into the relational model, making further integration among spreadsheets possible. Some extraction systems require explicit sheet-specific user-provided rules [2, 11], which might yield good results for a single spreadsheet. But they are not feasible for our setting: the corpus is large and users are not aware of the target spreadsheets to be processed ahead of time. It is impractical to manually transform all of them to relations. Abraham and Erwig[1] and Cunha et al. [8] automatically infer some spreadsheet structure, but they cannot process hierarchical spreadsheets. This type of spreadsheet is commonplace and extracting metadata from such spreadsheets presents the central technical challenge of this paper. We will illustrate these challenges using an example of a Web spreadsheet downloaded from the U.S. Census Bureau.

Challenges - The spreadsheet in Figure 1 shows a spreadsheet about the smoking rate downloaded from the government's Statistical Abstract of the United States. ${ }^{1}$. Each row clearly represents a different configuration of the smoking rate; for example, 13.7 in the value region is the rate for people with constraints Male, White, 65 years and over in the attribute region, and it yields an annotating relational tuple at the bottom. But there are two main problems here. First, the spreadsheet only implicitly indicates which cells carry values versus attributes. Often a spreadsheet is a mix of attributes, values, and other elements such as titles and footnotes. These elements are not easily distinguished from each other. Second, the spreadsheet does not explicitly indicate which attributes describe which values. If the leftmost column is processed naïvely, rows 25,31 , and 37 will yield three tuples that have different smoking rates for 65 years and older. All three extracted tuples are incorrect, as none

\footnotetext{
${ }^{1}$ http://www.census.gov/compendia/statab/2012/tables/12s0204.xls
} 


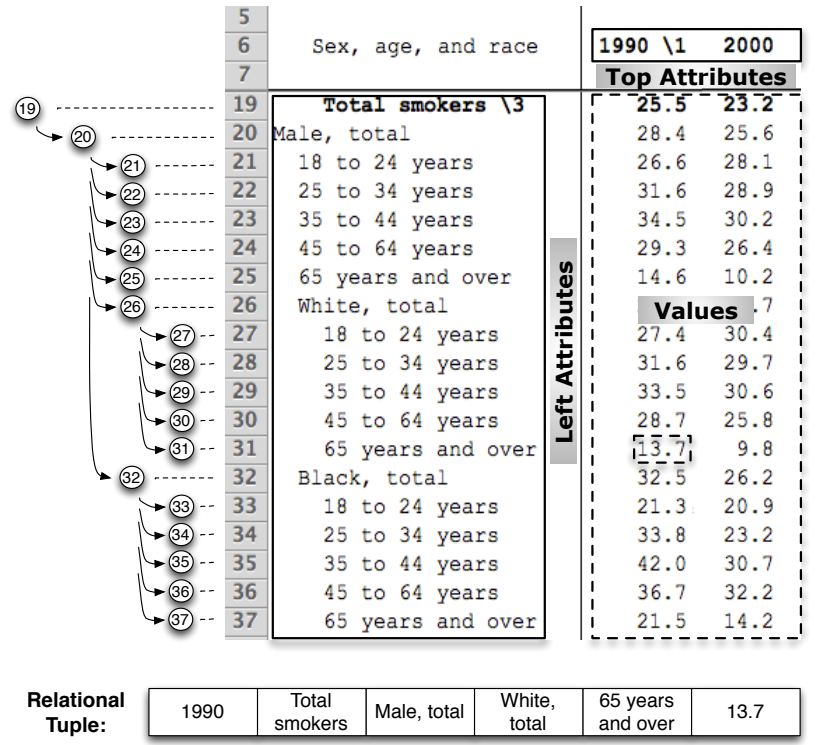

Figure 1: A portion of one spreadsheet from the U.S. Census Bureau.

will contain any mention of the attribute Male. In summary, Figure 1 shows a clean, high-quality spreadsheet, but extracting relational data from it requires us to: (1) detect attributes and values, (2) identify the hierarchical structure of left and top attributes, and (3) generate a relational tuple for each value in the spreadsheet.

Background - We implemented SEnBAzUru [5], a prototype spreadsheet database management system (SSDBMS). SENBAZURU focuses on data frame spreadsheets, which are one of the most popular types in the Web. Senbazuru searches a large number of Web crawl spreadsheets, and it automatically transforms spreadsheets into relations while allowing users to fix the extraction errors effectively and efficiently. Finally, it supports selection queries on the resulting relations and join queries to integrate arbitrary spreadsheets. In summary, SENBAZURU is able to extract relational information from a large number of Web spreadsheets, which makes it possible to directly manage spreadsheet data in databases; doing so also opens up opportunities for data integration among spreadsheets and with many other relational data sources.

Contributions - In this paper, we present the first automatic, domain-independent spreadsheet extractor, which is the first step in building SEnBAZuRu. First, we analyzed our Web crawl spreadsheets, 410,554 Microsoft Excel files from 51,252 distinct Internet domains. Our findings on those Web spreadsheets help us to better understand numerous spreadsheet usage scenarios on the Web and motivate our goal to design a practical SSDBMS. Second, our spreadsheet extractor automatically recognizes spreadsheet's layout, discovers the implicit metadata structure and emits relational data for a given spreadsheet. In contrast to previous research, our extraction does not require users to explicitly provide extraction rules. In our follow-up work, we will explore how to effectively and efficiently incorporate users' efforts to fix extraction errors, thus generating perfect relational tables from spreadsheets.

Applications - The central application goal of this work is a system that can combine spreadsheet data from many different sources, including the Web, intranets, and local storage. But there are a number of byproducts from our extraction procedure that could be useful for other semantic Web applications. For example, the procedure recovers a large amount of hierarchical metadata that is implicit in the spreadsheet and may not exist in a more formal database. Consider the organizational hierarchy of a small company; the management relationships among employees may not exist in a formal LDAP system but could plausibly be recovered from a stray spreadsheet. There may also be businessspecific hierarchies (e.g., families of industrial materials, or manufacturing stages) that are otherwise not recorded. These hierarchies are critical for our integration and search application but could also be useful on their own merit when combined with other semantic tools.

Organization - The paper is organized as follows.

- We describe a general survey of spreadsheet data practices based on 410,554 spreadsheets we downloaded from a general Web crawl (Section 2).

- We present our domain-independent extractor that obtains relational tuples from raw spreadsheets without any human intervention (Section 3).

- We evaluate the system's accuracy on a random sample of 100 Web hierarchical spreadsheets. We find that our methods can accurately obtain relational tuples from a spreadsheet; compared to a standard technique, our method reduces the amount of work a human must perform between $72 \%$ and $92 \%$ on average (Section 4 ).

In Section 5, we differentiate our work from the previous related work. Finally, we conclude and discuss the future work in Section 6.

\section{THE WEB SPREADSHEET CORPUS}

We obtained 410,554 Microsoft Excel files from 51,252 distinct Internet domains from our Web crawl. We call this collection the WEB dataset. We located the spreadsheets by looking for Excel-style file endings among roughly ten billion URLs in the ClueWeb09 Web crawl [6]. However, to the best of our knowledge, there is no other study that has surveyed a large number of spreadsheets in the Web. Therefore in this section, we aim to create the first portrayal of the WEB dataset in order to design our spreadsheet extraction pipeline. But first, we introduce notation to describe spreadsheets.

\subsection{Spreadsheet Notations}

In this paper, we focus on a two-part spreadsheet structure that we call a data frame. Data frame spreadsheets represent a type of spreadsheet, and this structure consists of two semantic components: a block of numeric values as a value region and accompanied attribute or metadata regions on the top or to the left. For attributes on the right, we treat them as an extension of left attributes. For example, Figure 1 shows a data frame with a value region indicated by the dashed rectangle and attribute regions on the top and to the left indicated by the solid rectangles.

A hierarchical spreadsheet is a data frame spreadsheet with either a hierarchical left attribute region or a hierarchical top attribute region. An example of a hierarchical left region can be found in Figure 1, and an example of hierarchical top region is shown in Figure 5. In contrast, flat 


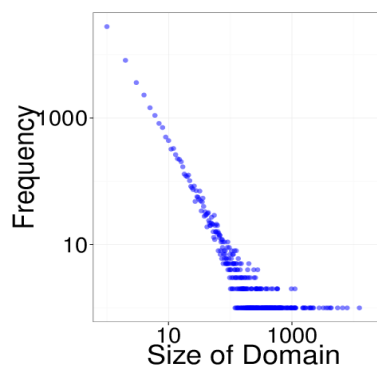

Figure 2: The distribution of Web spreadsheets.

\begin{tabular}{|l|cc|ccc|}
\hline \hline Domain & \# files & \% total & data frame & h-top & h-left \\
\hline \hline www.bts.gov & 12435 & $3.03 \%$ & $99 \%$ & $30 \%$ & $40 \%$ \\
\hline www.census.gov & 7862 & $1.91 \%$ & $94 \%$ & $72 \%$ & $70 \%$ \\
\hline www.stat.co.jp & 6633 & $1.62 \%$ & x & x & x \\
\hline www.bankofengland.co.uk & 5520 & $1.34 \%$ & $98 \%$ & $77 \%$ & $35 \%$ \\
\hline www.ers.usda.gov & 4328 & $1.05 \%$ & $95 \%$ & $77 \%$ & $70 \%$ \\
\hline www.agr.gc.ca & 4186 & $1.02 \%$ & $87 \%$ & $77 \%$ & $81 \%$ \\
\hline www.wto.org & 3863 & $0.94 \%$ & $96 \%$ & $61 \%$ & $77 \%$ \\
\hline www.doh.wa.gov & 3579 & $0.87 \%$ & $81 \%$ & $53 \%$ & $64 \%$ \\
\hline www.nsf.gov & 2770 & $0.67 \%$ & $96 \%$ & $53 \%$ & $76 \%$ \\
\hline nces.ed.gov & 2177 & $0.53 \%$ & $98 \%$ & $55 \%$ & $92 \%$ \\
\hline average & $\mathbf{5 3 3 5}$ & $\mathbf{1 . 3 0 \%}$ & $\mathbf{9 3 . 7 8 \%}$ & $\mathbf{6 1 . 6 7 \%}$ & $\mathbf{6 7 . 3 3 \%}$ \\
\hline \hline
\end{tabular}

Figure 3: The top 10 domains in our Web spreadsheet corpus. h-top and h-left are percentages of spreadsheets with a hierarchical top or left region. spreadsheets refer to those spreadsheets without any hierarchical structure.

For each value in the value region, there is usually (but not necessarily) at least one annotating string in the top and left regions, generating a relational tuple. For example, in Figure 1, the value 13.7 is annotated by 65 years and over, White total, Male total, and Total smokers in the left region and by 1990 in the top region, yielding a relational tuple.

\subsection{Web Spreadsheets Survey}

To better design our extraction system, we answer the following critical questions about the general properties of the Web spreadsheets and the popularity of the data frame spreadsheets on the Web:

1. Where are those Web spreadsheets from? The Web spreadsheets cover a huge range of topics and show wide variance in cleanliness and quality. Most of the spreadsheets are statistical data, with a heavy emphasis on government, finance, transportation, etc. We are also interested in the distribution of the spreadsheets from different Internet domains. Figure 3 shows the top 10 Internet domains that host the largest number of spreadsheets in the WEB corpus. Nine of the top 10 domains are sites run by the U.S., Japanese, UK, or Canadian governments. Figure 2 shows the distribution of spreadsheets among hosting domains. We rank the domains according to the size of their hosting spreadsheets in descending order. The plot indicates that the spreadsheets follow a strongly skewed distribution, with a large number of spreadsheets from relatively few domains and with a large number of domains hosting relatively few spreadsheets.

2. How many of the Web spreadsheets consist of data frame structures? To better understand the structure of the WEB spreadsheets, we randomly chose 200 samples and asked a human expert to mark their structures. We found $50.5 \%$ of the spreadsheets consist of data frame components and $32.5 \%$ have hierarchical top or left attributes. The other $49.5 \%$ non-data frame spreadsheets belong to the following categories: $22.0 \%$ are Relation spreadsheets that can be converted to the relational model almost trivially (we can simply translate each spreadsheet column into a relational table column and translate each spreadsheet row into a relational tuple); $10.5 \%$ are Form spreadsheets that are not for data storage and are designed to be filled by a human; $3.5 \%$ are Diagram spreadsheets for visualization purposes, and they are often data-intensive without any schema information; and 3\% are List spreadsheets that consist of nonnumeric tuples. The $10.5 \%$ Other spreadsheets are schedules, syllabi, scorecards, or other files whose purpose is unclear.
Although there are a variety of categories of spreadsheets on the Web, in this paper, we only focus on data frame spreadsheets.

3. How many of the Web spreadsheets are hierarchical like the example shown in Figure 1? Are those hierarchical spreadsheets spread uniformly across the $\boldsymbol{W e b}$ ? As just mentioned, $32.5 \%$ of the 200 sample Web spreadsheets have hierarchical top or left attributes in a data frame. To better understand how the hierarchical spreadsheets are distributed in different domains, we randomly selected 100 spreadsheets from each of the top 10 domains, yielding 900 spreadsheets in total. ${ }^{2}$ Figure 3 shows the fraction of spreadsheets with data frames or hierarchical attributes in the top 10 domains. The ratios are much higher than the fractions we obtained from the general Web sample. We also randomly selected 100 spreadsheets from domains hosting fewer than 10 spreadsheets. We found $19 \%$ with data frame structures, $4 \%$ of which have hierarchical top attributes and $6 \%$ of which have hierarchical left attributes. These results suggest that the number of hierarchical spreadsheets differs greatly by domain and may be linked to the domain's popularity or degree of professionalism. Computing the exact distribution of hierarchical spreadsheets among domains would be useful but requires a huge amount of labeled data; we will explore this question in future work. Even without computing that distribution, we have found a huge number of hierarchical spreadsheets: $32.5 \%$ of all spreadsheets on the Web and more than 60\% in popular domains. Therefore, to extract relational data from spreadsheets, we believe our system must process hierarchical-style metadata.

Overall, we observe that: (1) the Web contains a huge variety of spreadsheets from a large range of data sources, and (2) the data frame spreadsheets, especially the hierarchical ones, are highly popular in the Web. Therefore, to design a system to extract relational data from spreadsheets, the system should be able to process data frame spreadsheets, especially those hierarchical-style spreadsheets.

\section{SYSTEM PIPELINE}

In this section, we describe our spreadsheet extraction pipeline. The goal of the extraction pipeline is to create a relational model of the data embedded in data frame spreadsheets: it takes in a data frame spreadsheet and emits relational tuples. It should be able to work on both flat and hierarchical spreadsheets (we treat flat spreadsheets as a

\footnotetext{
${ }^{2}$ www.stat.co.jp is excluded because it is in Japanese.
} 


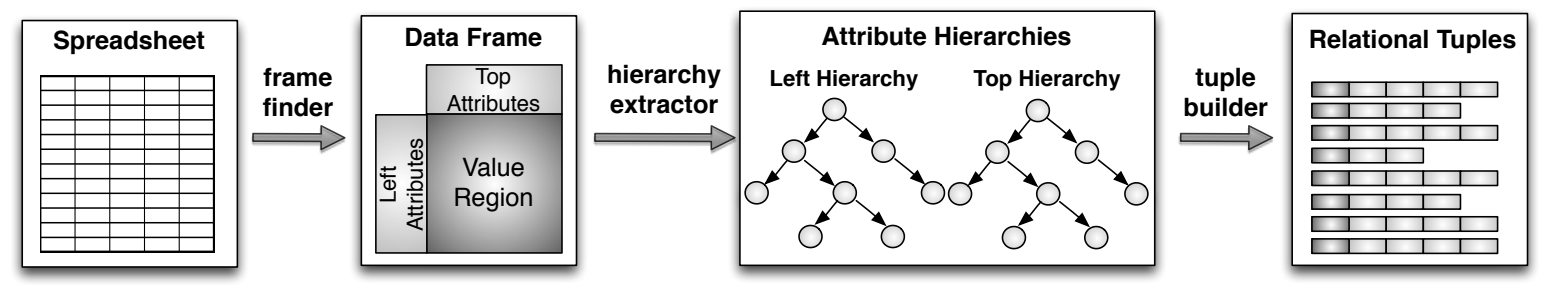

Figure 4: The system pipeline to process a single spreadsheet.

special case of hierarchical ones). As shown in Figure 4, the extraction pipeline consists of three components: the frame finder, the hierarchy extractor, and the tuple builder. The frame finder identifies the data frames, locating attribute regions and value regions. The hierarchy extractor recovers the hierarchical metadata from spreadsheets, and the tuple builder generates a relational tuple for each value in the value region.

Notice that the extraction pipeline does not explicitly distinguish data frame spreadsheets from non-data frame spreadsheets. We assume that given a spreadsheet, if the pipeline could process the spreadsheet and output a set of relational tuples in a good quality, then the spreadsheet is a data frame spreadsheet; otherwise it is not. Of course, this approach might yield false positive data frame spreadsheets. However, a post-processing stage could be added to the end of the pipeline to further filter the non-data frame spreadsheets. We skip details of this post-processing component because it is beyond the scope of this paper.

\subsection{Frame Finder}

The frame finder identifies the value region and the top and left attribute regions. It receives a raw spreadsheet as input and emits geometric descriptors of the data frame's three rectangular regions. We define the problem as follows:

DeFinition 1. (Frame Finder) Let a spreadsheet be a grid of cells $\mathbf{c}=\left\{c_{i j}\right\}$, where $i$ represents the row index and $j$ represents the column index. The frame finder assigns each cell $c_{i j} \in \mathbf{c}$ with a label $l_{i j} \in \mathrm{L}=\{$ top, left, value, other\}, where top represents top attributes, left represents left attributes, value represents values, and other represents everything else.

To simplify the problem, we assume that the structure of the spreadsheets has the following property: there may be multiple data frames in a spreadsheet, but they only stack in the vertical dimension. In fact, we found less than $2 \%$ of the 900 spreadsheets in Figure 3 violate the assumption. This assumption allows us to treat data frame-finding as a problem of row labeling. Therefore, we start with the row labeler task, which assigns each row in a spreadsheet to one of the following four categories: title, header, data, or footnote. The label title represents a spreadsheet title, header represents a row that contains top attributes only, data represents a row that contains left attributes or values, and footnote is information that annotates the main contents. As in Figure 1, rows 5-7 are labeled header and rows 19-37 are labeled data. A formal definition is as follows:

Definition 2. (Row Labeler) Let $\mathbf{r}=\left\{r_{1}, r_{2}, \ldots, r_{N}\right\}$ be a set of variables representing the non-empty rows in a spreadsheet. The row labeler assigns each $r_{i} \in \mathbf{r}$ with a label $l_{i} \in L=\{$ title, header, data, footnote $\}$.

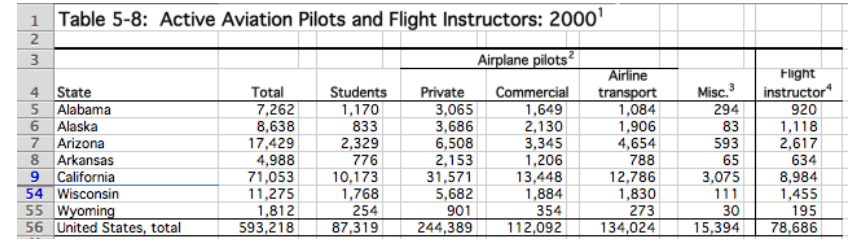

Figure 5: An example of hierarchical top attributes.

We observe the following two types of signals that the row labeler should use to automatically assign semantic labels to each non-empty row: (1) the properties of each non-empty row indicate its semantic label, such as its fonts and keywords; and (2) the labels assigned to adjacent rows are highly related. For example, if we know the current row is a header row, it is highly probable that the next row is a header or data row. Therefore, we employ an approach based on a linear-chain, conditional random field (CRF) [13] to exploit these two types of signals. Pinto et al. [16] used linear-chain CRFs to obtain labels for textual tables in government statistical text reports. We also use the linear-chain CRFs to obtain the semantic labels for each row of a spreadsheet, and our training and inference procedure is the same. However, with the access to spreadsheet APIs, we are able to build the CRFs with a richer set of features, such as the alignment and indentation information that is hard to obtain from plain text reports. Our extraction features fall into two main categories: layout features test visual characteristics of a row, and textual features test the contents of the row. The details of the features can be found in Appendix A.

Once we have labels for each row in a spreadsheet, we can construct the correct data frame regions. The vertical extent of a value region is described by the set of rows marked data, and its horizontal extent is determined by finding regions of numeric values. The top attribute region is delimited by all header rows, and the left attribute region is everything to the left of the value region.

\subsection{Hierarchy Extractor}

The hierarchy extractor recovers the attribute hierarchies. This step receives a data frame with top and left regions as input and emits hierarchies as output: one for left and one for top. These trees describe the hierarchical annotation relationship among attributes in the top and left regions. For example, in Figure 1, row 31 is annotated by attributes at rows 26, 20, and 19. An example of a top hierarchy can be found in Figure 5, where the attribute Airplane pilots annotates the attribute Airline transport. Now we formally describe the problem of recovering the attribute hierarchy for a single region as follows: 
Definition 3. (Hierarchy Extractor) Let $A=\left\{a_{1}, \ldots, a_{N}\right.$, root $\}$ represent the set of cells in an attribute region. Given $a_{i}, a_{j} \in A$, we say $\left(a_{i}, a_{j}\right)$ is a ParentChild pair if $a_{i}$ is a parent attribute of $a_{j}$ in the hierarchy. The hierarchy extractor identifies all the ParentChild pairs in the attribute region.

For example, consider the hierarchy on the left of Figure 1 where each node represents an attribute in the corresponding row. $(20,26)$ is a ParentChild pair, while $(20,31)$ and $(24,25)$ are not. For a top hierarchy as shown in Figure 5, (Airplane pilots, Airline transport) is a ParentChild pair.

The goal of the hierarchy extractor is to find all such ParentChild pairs in an attribute region, thus describing a hierarchical tree. It may seem that a simple heuristic can recover the annotation hierarchies, but in practice, a correct heuristic is extremely hard to obtain and to generalize to a large number of spreadsheets. As in Figure 1, a simple heuristic, such as the indentations of left attributes, may identify some ParentChild pairs but fails to recognize (19, 20) as a ParentChild pair. Therefore, to obtain a correct hierarchy, we need to use a probabilistic method to exploit a variety of signals. In the rest of the section, we discuss our proposed two classification-based algorithms.

\subsubsection{Algorithm 1: Classification}

To obtain all the ParentChild pairs in an attribute region, our classification-based approach first generates a series of ParentChild candidates and then finds all the true ParentChild pairs through classification.

A straightforward way to generate ParentChild candidates for an attribute region $A=\left\{a_{1}, \ldots, a_{N}\right.$, root $\}$ is to create a $\left(a_{i}, a_{j}\right)$ for each $a_{i}, a_{j} \in A$. This simple method will yield thousands of nodes for every single attribute region $A$ with $N$ attributes. The size of created candidates potentially could be very large, but in practice, we can greatly shrink the number by leveraging our observations on the spreadsheets to filter the unlikely candidates.

For left attributes, given a ParentChild pair candidate $\left(a_{i}, a_{j}\right)$ in a left attribute region, we observe that a candidate in two cases is not likely to be a ParentChild pair.

1. The formatting styles of the parent and child attributes are the same. We believe that formatting style of an attribute cell is a strong indication of the hierarchical structure. Given a ParentChild candidate $\left(a_{i}, a_{j}\right), a_{i}$ and $a_{j}$ are determined to share the same formatting style or not based on a predefined style feature vector. The style feature vector for each attribute, style(a), is a combination of the unary extraction features shown in Table 5 and the attribute's font size and indentation information. If we have $\operatorname{style}\left(a_{i}\right)=\operatorname{style}\left(a_{j}\right)$, then $\left(a_{i}, a_{j}\right)$ is not likely to be a true ParentChild pair. For example in Figure 1, the left attribute at row 23 has the same formatting style as the one at row 21: style $(23)=$ style $(21)$, and so we do not consider this a ParentChild candidate $(21,23)$.

2. We prioritize the similar ParentChild pairs which are geometrically close to each other in the spreadsheet. For example, as shown in Figure 1, we create two ParentChild pair candidates $(32,33)$ and $(26,33)$, and the two candidates are considered similar because 32 (Black, total) and 26 (White, total) are both talking about race. As a result, we only keep the pair $(32,33)$ with attributes geometrically closer and remove the ParentChild candidate $(26,33)$.

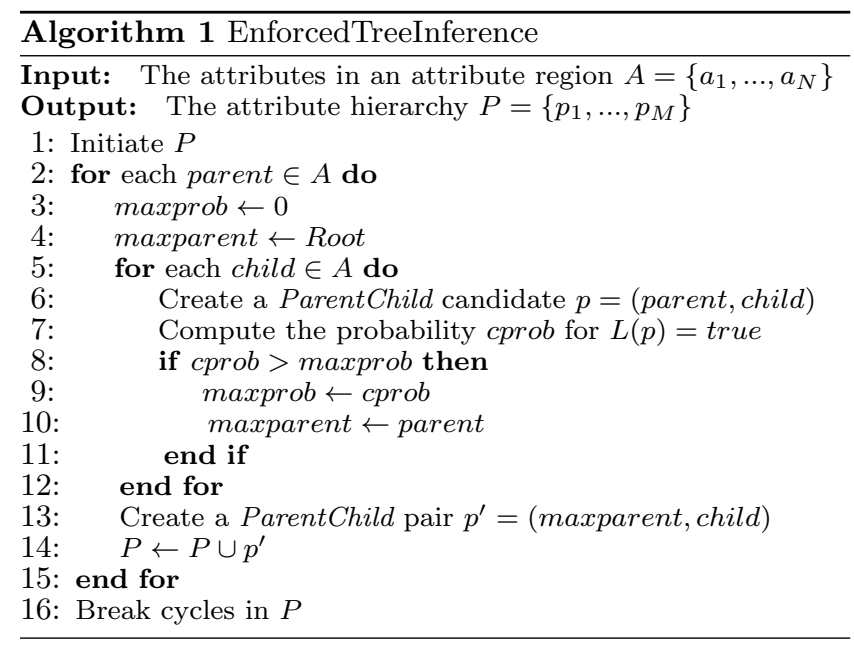

For top attributes, given a ParentChild candidate $\left(a_{i}, a_{j}\right)$ in a top attribute region, we believe $\left(a_{i}, a_{j}\right)$ is not likely to be a true ParentChild pair if the row of the child attribute $a_{j}$ is lower than the row of the parent attribute $a_{i}$. Of course, more heuristics could be found to further filter the unlikely ParentChild candidates, but in practice, this simple rule is already effective enough to greatly shrink the size of the created ParentChild candidates.

Now we formally describe the classification process: given a set of ParentChild pair candidates $P=\left\{\left(a_{i}, a_{j}\right)\right\}$ in an attribute region, the classifier assigns each $p=\left(a_{i}, a_{j}\right) \in P$ with a label from $L=\{$ true, false $\}$ s.t. the predicted ParentChild pairs $\left\{\left(a_{i}, a_{j}\right) \mid L\left(a_{i}, a_{j}\right)=\right.$ true $\}$ construct an attribute hierarchy in the given attribute region. If the classification is entirely correct, the produced ParentChild pairs represent a tree. However any error in the classification might yield an inaccurate result.

Our classification-based method exploits a variety of signals in a spreadsheet to extract attribute hierarchies. For left and top attributes, we use a different set of features. For left attributes, the classifier utilizes two types of features: unary features and binary features; for top attributes, we mainly utilize layout features. The features we used for both left and top regions are discussed in detail in Appendix B.

\subsubsection{Algorithm 2: Enforced-tree Classification}

One weakness of the classification-based approach is that it does not guarantee that the emitted ParentChild pairs construct a tree. Thus, our second proposed technique is enforced-tree classification, which embeds simple heuristics into the classification results to ensure the produced pairs construct a strict hierarchical tree. Of course, in a tree structure, each node has only one parent (except the root). Thus for each attribute, we select the one with the maximal probability as its parent attribute. We obtain the probability associated with each ParentChild pair during the classification. This one-parent constraint does not guarantee that the output will be a tree, as cycles may still exist in the results. We then iteratively break cycles by deleting the pairs with the minimal probability until there are no more cycles in the output. We assume one's parent attribute is Root by default. Therefore, the two steps, the one-parent constraint and the breaking-cycles, enforce the classification results to generate a strict tree. The classifier uses the same 


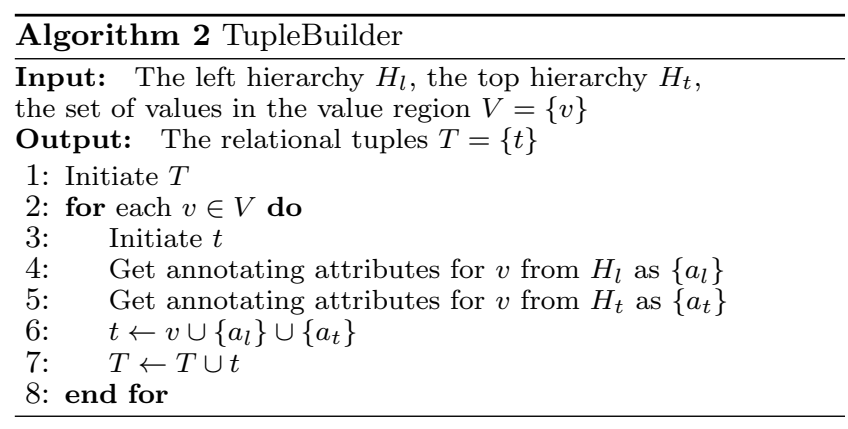

set of features as the classification method, and the details of the algorithm are shown in Algorithm 1.

\subsection{Tuple Builder}

The tuple builder is straightforward, as long as the previous steps are accurate. We generate a relational tuple for each value in the value region, annotating each one with relevant attributes from the attribute hierarchies. For example, Figure 1 shows the full six-field tuple we want to recover for the highlighted value 13.7. The tuple builder is also algorithmically straightforward. It processes the extracted attribute hierarchies and the value region to generate a series of relational tuples. As described in Algorithm 2, for each value $v$, we find the its annotating attributes along the path to the root in the attribute hierarchies for both left and top attribute regions. The tuple builder relies entirely on the frame finder and hierarchy extractor for correctness.

\section{EXPERIMENTS}

We can now quantify the performance of the system by evaluating its individual components. In particular, we present the performance of the frame finder and the hierarchy extractor. We do not directly evaluate the tuple builder because it entirely relies on the correctness of the hierarchy extractor, and it will yield the ideal results as long as it receives accurate hierarchies.

In the following experiments, we use 100 random hierarchical data frame spreadsheets (data frame spreadsheets with hierarchical top attributes or hierarchical left attributes). We obtained this dataset by randomly sampling spreadsheets from WEB and only keeping those with a hierarchical data frame. For top, the average hierarchy depth of the dataset is 2.14 , with a maximum depth of 5 ; for left, the average hierarchy depth is 2.61 , with a maximum depth of 9. The training and testing procedures for both the row labeler and the hierarchy extractor are as follows: we randomly split the dataset into equal-sized training and testing sets, repeating this process 10 times. Then we report the average Precision, Recall, and F1 measure for each class. We also present the mean and standard deviation (std) for errors per sheet, which is defined as follows:

Definition 4. (Errors per sheet) A classification task produces two types of errors, false positive $(f p)$ and false negative $(f n)$, on $N$ spreadsheets. We define errors per sheet:

$$
\text { Errors }_{\text {sheet }}=(f p+f n) / N
$$

For the experiment setup, we used several open-source packages: for frame finder, our CRFs were implemented on $\mathrm{CRF}++[7]$; for hierarchy extractor, we used the SVM

\begin{tabular}{|c|c|ccc|cc|}
\hline \hline \multirow{2}{*}{} & \multicolumn{3}{|c|}{ Performance } & \multicolumn{2}{c|}{ Errors } \\
\cline { 3 - 7 } \multicolumn{2}{c|}{} & Precision & Recall & F1 & Mean & Std \\
\hline \hline \multirow{2}{*}{ title } & Base-CRF & 0.561 & 0.605 & 0.582 & 3.534 & 4.532 \\
& Full-CRF & 0.818 & 0.734 & 0.774 & 0.872 & 0.150 \\
\hline \multirow{2}{*}{ header } & Base-CRF & 0.624 & 0.606 & 0.615 & 2.348 & 0.621 \\
& Full-CRF & 0.812 & 0.740 & 0.774 & 1.316 & 0.343 \\
\hline \multirow{2}{*}{ data } & Base-CRF & 0.995 & 0.970 & 0.982 & 6.526 & 5.239 \\
& Full-CRF & 0.995 & 0.993 & 0.994 & 1.528 & 0.330 \\
\hline \multirow{2}{*}{ footnote } & Base-CRF & 0.550 & 0.786 & 0.647 & 4.208 & 3.414 \\
& Full-CRF & 0.843 & 0.826 & 0.834 & 1.208 & 0.223 \\
\hline \hline
\end{tabular}

Table 1: Performance of the row labeler.

\begin{tabular}{|c|c|ccc|cc|}
\hline \hline \multicolumn{2}{|c|}{} & \multicolumn{3}{|c|}{ Performance } & \multicolumn{2}{c|}{ Errors } \\
\cline { 3 - 7 } \multicolumn{2}{|c|}{} & Precision & Recall & F1 & Mean & Std \\
\hline \hline \multirow{2}{*}{ top } & SVM & 0.921 & 0.918 & 0.919 & 1.834 & 0.398 \\
& EN-SVM & 0.920 & 0.920 & 0.920 & 1.829 & 0.395 \\
\hline \multirow{2}{*}{ left } & SVM & 0.852 & 0.700 & 0.769 & 19.554 & 5.107 \\
& EN-SVM & 0.811 & 0.811 & 0.811 & 16.154 & 4.332 \\
\hline \hline
\end{tabular}

Table 2: Performance of the hierarchy extractor.

library from the LIBSVM package [4] and the Weka package [10] for the logistic regression and naive Bayes method.

\subsection{Frame Finder}

We now evaluate the performance of the frame finder described in Section 3.1 by evaluating the row labeler. Our experiment spreadsheets contain 27,531 non-empty rows that are correctly assigned with semantic labels by a human expert. In [16], CRFs were used to label the lines of tables in plain-text government statistical reports using textual features. Our row labeler also uses CRFs but incorporates richer features: both textual and layout features. The layout features, such as bold font and alignment, are hard to obtain from plain text but accessible in spreadsheets through the Python xlrd library. Therefore, we compare two CRFs with different sets of features: Base-CRF for textual features and Full-CRF for textual + layout features.

As shown in Table 1, Full-CRF performs significantly better than Base-CRF on all the metrics, including precision, recall, and errors per sheet. According to precision and recall, both methods do a decent job of predicting all the labels, but they show a large difference in the number of errors, especially for the label data. For data, the two methods have very close precision and recall, but Base-CRF produces many more errors than Full-CRF. This occurs because of the huge number of data rows in the dataset, and a small difference in the F1 measure will make a big difference to the absolute number of errors. Overall, Table 1 shows that the Full-CRF method is superior to the baseline Base-CRF method and can work effectively as a part of the system. Full-CRF predicts each category fairly accurately, and the number of errors produced by Full-CRF is tolerable for all the labels, with about one error per sheet.

\subsection{Hierarchy Extractor}

We evaluate the performance of the hierarchy extractor discussed in Section 3.2 by measuring its accuracy in retrieving correct ParentChild pairs from a spreadsheet. The hierarchical metadata in spreadsheets is unique, and we are not aware of any previous method to automatically extract such hierarchical metadata. Therefore, we create a baseline approach Human to ask a user to manually enumerate all the ParentChild pairs in an attribute region, which is exactly what the hierarchy extractor infers automatically. We first evaluate the performance of our two approaches: 


\begin{tabular}{|c|c|c|}
\hline \hline \multicolumn{2}{|c|}{} & Repairs \\
\hline \hline \multirow{3}{*}{ top } & Human & 22.469 \\
& SVM & 1.834 \\
& EN-SVM & 1.829 \\
\hline \multirow{3}{*}{ left } & Human & 58.598 \\
& SVM & 19.554 \\
& EN-SVM & 16.154 \\
\hline \hline
\end{tabular}

Table 3: User repair \# for the hierarchy extractor.

SVM for classification and EN-SVM for enforced-tree classification. We then compare our two methods with the baseline method, Human, on the metric user repair \#.

Definition 5. (User Repair \#) We assume that a user reviews every ParentChild pair candidate with an assignment, true or false, in an attribute region. User repair \# is the number of assignments the user must modify in order to obtain the correct hierarchy.

For SVM and EN-SVM, user repair \# equals errors per sheet in a given attribute region. For Human, user repair \# is the total number of true ParentChild pairs in an attribute region (we assume that all the ParentChild pair candidates are labeled false by default).

Table 2 shows the performance of our two methods. As seen in Table 2, EN-SVM performs the best, especially on left. Note that for left, EN-SVM has a higher recall than SVM but a slightly lower precision. The reason is that given an attribute, all the ParentChild candidates containing its parent attribute may be labeled false by the classifier, and then the attribute will not have any parent attribute. But EN-SVM is able to recover its parent attribute by selecting the most probable ParentChild pair from the false group. Table 3 presents the user repair \# for the three methods and shows that both SVM and EN-SVM require a much smaller number of user repairs than Human. We also tried logistic regression and naive Bayes for classification. Overall, SVM is comparable to logistic regression but performs the best of the three. Therefore, we conclude that our method EN-SVM is superior to the baseline Human, as EN-SVM predicts the ParentChild fairly accurately and it beats Human on user repair \#.

One limitation of our system lies in the fact that the absolute number of required repairs on left is not trivial. According to Table 3, the number of repairs on top is almost negligible, but not on left. We will try to reduce the user burden even further in future work.

\section{RELATED WORK}

There are three main types of existing approaches to transform spreadsheet data into databases. The first is a schemabased approach. For spreadsheet data already in a relational format, traditional schema mapping systems, such as Clio [9] and Clip [17], could potentially be used to convert the spreadsheet data into databases by specifying the source and target attribute mapping. The second is a rule-based approach [11], which requires explicit user-provided conversion rules. Finally, a visualization-based approach provides users with an interactive visualization interface to convert or manage the underlying data $[12,18,19,21]$. However, these existing approaches all suffer from two common drawbacks: (1) it is challenging to handle hierarchical spreadsheets; (2) the transformation process cannot be accomplished automatically: most of the approaches require users to learn a new language or predefined operators to describe the transformation rules. In fact, the work of Abraham and Erwig [1] and Cunha et al. [8] are the most similar to ours. The former attempts to infer spreadsheet metadata, but they do not address the hierarchical structures in spreadsheets. The latter tries to convert spreadsheets into relational databases, but their primary technical focus is to address the lack of data normalization in spreadsheets that use very conventional layouts. In addition, the approach does not address hierarchical spreadsheets.

There is other research on spreadsheets attempting to build database-like operators on a spreadsheet-style interface [14, 22, 23, 25], but these systems cannot be directly used to manage the large number of spreadsheets that already exist on the Web. The QueryByExcel project [23, $24,25]$ uses a spreadsheet as a front end of the relational database. It translates Excel formulas using an extension of SQL relational operations and performs on RDBMS tables. Liu et al. [14] implemented an extended set of database functions operating on spreadsheets, and the operations are executed by a classic database engine in the background. Tyszkiewicz [22] also attempted to combine SQL with spreadsheets but implemented the functionality inside spreadsheets instead of using an additional database engine.

\section{CONCLUSIONS AND FUTURE WORK}

We have described a domain-independent spreadsheet extraction system for converting spreadsheet data into relational tuples. Our system consists of three components that detect the structure of a spreadsheet, extract hierarchical metadata, and generate relational tuples. Our experiments show that our proposed methods are superior to the baseline approaches and can work effectively as a part of the whole framework. As a result, the system can help bring relational-style data management techniques to the mass of data currently locked in spreadsheets. The extraction procedure also emits various semantic byproducts in the form of hierarchies that could be useful in a range of other applications, such as schema integration and design tools.

One area of future work lies in how to best incorporate manual repairs to further reduce users' burden. Another lies in the integration application itself; extraction is a necessary first step, and we have not yet rigorously addressed search ranking quality and join findability in the end user tool.

\section{ACKNOWLEDGMENTS}

This project is supported by National Science Foundation grants IIS-1054913 and IIS-1064606, as well as gifts from Dow Chemical, Yahoo!, and Google. Special thanks to Robert Vogel for advice and assistance.

\section{REFERENCES}

[1] R. Abraham and M. Erwig. Ucheck: A spreadsheet type checker for end users. J. Vis. Lang. Comput., 18(1):71-95, 2007.

[2] Y. Ahmad, T. Antoniu, S. Goldwater, and S. Krishnamurthi. A type system for statically detecting spreadsheet errors. In $A S E$, pages 174-183, 2003.

[3] P. Blattner and L. Stewart. Microsoft excel 2000 functions in practice. QUE, 1999.

[4] C.-C. Chang and C.-J. Lin. LIBSVM: A library for support vector machines. ACM Transactions on Intelligent Systems and Technology, 2:27:1-27:27, 2011.

[5] Z. Chen, M. Cafarella, J. Chen, D. Prevo, and J. Zhuang. Senbazuru: A prototype spreadsheet database management system. VLDB Demo, 2013. 
[6] 2009. ClueWeb09, http://lemurproject.org/clueweb09.php.

[7] 2009. http://crfpp.sourceforge.net.

[8] J. Cunha, J. Saraiva, and J. Visser. From spreadsheets to relational databases and back. In PEPM, pages 179-188, 2009.

[9] A. Fuxman, M. A. Hernández, C. T. H. Ho, R. J. Miller, P. Papotti, and L. Popa. Nested mappings: Schema mapping reloaded. In $V L D B$, pages $67-78,2006$.

[10] M. Hall, E. Frank, G. Holmes, B. Pfahringer, P. Reutemann, and I. H. Witten. The weka data mining software: an update. SIGKDD Explor. Newsl., 11(1):10-18, Nov. 2009.

[11] V. Hung, B. Benatallah, and R. Saint-Paul. Spreadsheet-based complex data transformation. In $C I K M$, pages 1749-1754, 2011

[12] T. Igarashi, J. D. Mackinlay, B.-W. Chang, and P. Zellweger. Fluid visualization for spreadsheet structures. In $V L$, pages 118-125, 1998.

[13] J. Lafferty, A. McCallum, and F. Pereira. Conditional random fields: Probabilistic models for segmenting and labeling sequence data. In ICML, pages 282-289, 2001.

[14] B. Liu and H. Jagadish. A spreadsheet algebra for a direct data manipulation query interface. In $I C D E$, pages 417-428, 2009.

[15] M. Nahm and J. Zhang. Operationalization of the ufurt methodology for usability analysis in the clinical research data management domain. Journal of Biomedical Informatics, 42(2):327-333, 2009

[16] D. Pinto, A. McCallum, X. Wei, and W. B. Croft. Table extraction using conditional random fields. In SIGIR, pages 235-242, 2003

[17] A. Raffio, D. Braga, S. Ceri, P. Papotti, and M. A. Hernández. Clip: a visual language for explicit schema mappings. In $I C D E$, pages 30-39, 2008.

[18] V. Raman and J. M. Hellerstein. Potter's wheel: An interactive data cleaning system. In $V L D B$, pages 381-390, 2001

[19] R. Rao and S. K. Card. The table lens: merging graphical and symbolic representations in an interactive focus + context visualization for tabular information. In $C H I$, pages $318-322$, 1994

[20] J. Simon. Excel 2000 in a nutshell. O'Relly Media, 2000.

[21] M. Spenke, C. Beilken, and T. Berlage. Focus: The interactive table for product comparison and selection. In $U I S T$, pages 41-50, 1996

[22] J. Tyszkiewicz. Spreadsheet as a relational database engine. In SIGMOD, pages 195-206, 2010.

[23] A. Witkowski, S. Bellamkonda, T. Bozkaya, G. Dorman, N. Folkert, A. Gupta, L. Sheng, and S. Subramanian. Spreadsheets in rdbms for olap. In SIGMOD Conference, pages $52-63,2003$

[24] A. Witkowski, S. Bellamkonda, T. Bozkaya, N. Folkert, A. Gupta, L. Sheng, and S. Subramanian. Business modelling using sql spreadsheets. In $V L D B$, pages 1117-1120, 2003.

[25] A. Witkowski, S. Bellamkonda, T. Bozkaya, A. Naimat, L. Sheng, S. Subramanian, and A. Waingold. Query by excel. In $V L D B$, pages 1204-1215, 2005.

\section{APPENDIX}

\section{A. FRAME FINDER FEATURES}

The features we used in the frame finder include layout and textual features. Each of the features is a binary function, taking in a given row in a spreadsheet as the input and emitting a $0 / 1$ Boolean value as the output. The features attempt to test whether the properties of a row are an indication of a certain category in $\{$ title, header, data, footnote $\}$. The features are listed in Table 4.

\section{B. HIERARCHY EXTRACTOR FEATURES}

Left Attributes - For left attributes, given a ParentChild pair candidate $\left(a_{i}, a_{j}\right)$, we employ a set of features to characterize its properties, thus determining whether it is a true ParentChild pair. The testing features include unary features and binary features, as shown in Table 5. The unary features apply on each of the child and parent attributes, and the binary features apply on the attribute pair.
Top Attributes - For top attributes, given a ParentChild pair candidate $\left(a_{i}, a_{j}\right)$, we utilize a set of layout features to characterize the properties of the attribute pair, thus determining whether it is a true ParentChild pair. The features we used are shown in Table 6 .

\begin{tabular}{|l|l|}
\hline \hline \multicolumn{2}{|c|}{ Layout Features } \\
\hline 1 & Has a bold font cell \\
\hline 2 & Has a cell reaching the left bound \\
\hline 3 & Has a cell reaching the right bound \\
\hline 4 & Has a cell with indentations \\
\hline 5 & Has a center-aligned cell \\
\hline 6 & Has a left-aligned cell \\
\hline 7 & Has a merged cell \\
\hline 8 & Has only one column \\
\hline \multicolumn{2}{|c|}{ Textual Features } \\
\hline 1 & Contains colon \\
\hline 2 & Contains punctuations \\
\hline 3 & Has a cell with with a word count $>40$ \\
\hline 4 & Numeric cells within year range ratio $>0.6$ \\
\hline 5 & Row is blank \\
\hline 6 & With all words in lowercases \\
\hline 7 & With all words capitalized \\
\hline 8 & With all words starting with capitals \\
\hline 9 & With numeric cells ratio $>0.6$ \\
\hline 10 & With words starting with "table" \\
\hline \hline
\end{tabular}

Table 4: Extraction features for the frame finder.

\begin{tabular}{|l|l|}
\hline \hline \multicolumn{2}{|c|}{ Unary Extraction Features } \\
\hline 1 & Attribute has underline \\
\hline 2 & Attribute contains keywords like "total" \\
\hline 3 & Attribute contains colon \\
\hline 4 & Attribute is bold \\
\hline 5 & Attribute is center aligned \\
\hline 7 & Attribute is italic \\
\hline 8 & Attribute is numeric \\
\hline 9 & Attribute letters are all capitalized \\
\hline 10 & Is the last attribute \\
\hline \multicolumn{2}{|c|}{ Binary Extraction Features } \\
\hline 1 & Attribute pair is adjacent \\
\hline 2 & Attribute pair's indentation is equal \\
\hline 3 & Attribute pair's style is adjacent in the region \\
\hline 4 & Child's font size is smaller than parent's \\
\hline 5 & Child's indentation is greater than parent's \\
\hline 6 & Child's row index is greater than parent's \\
\hline 7 & Child's style is the same as the first attribute \\
\hline 8 & Has blank cells in the middle \\
\hline 9 & Has middle cell with indentation between the pair's \\
\hline 10 & Has middle cell with indentation larger than the pair's \\
\hline 11 & Has middle cell with indentation less than the pair's \\
\hline 12 & Has middle cell with style different from the pair's \\
\hline 13 & Has middle cell containing keywords like "total" \\
\hline 14 & Parent is the root \\
\hline \hline
\end{tabular}

Table 5: Extraction features for the hierarchy extractor on left attributes.

\begin{tabular}{|l|l|}
\hline \hline \multicolumn{2}{|c|}{ Layout Extraction Features } \\
\hline 1 & Child has no cell right above \\
\hline 2 & Child is at the uppermost header row \\
\hline 3 & Has a cell in the middle \\
\hline 4 & Parent cell covers child's column \\
\hline 5 & Parent is on the left of child \\
\hline 6 & Parent is on the right of child \\
\hline 7 & Parent is right above child \\
\hline 8 & Parent is the root \\
\hline \hline
\end{tabular}

Table 6: Extraction features for the hierarchy extractor on top attributes. 